\title{
Percepção dos idosos sobre as repercussões da pandemia por COVID-19 em sua
}

\section{saúde}

\author{
Elderly perception of the repercussions of the COVID-19 pandemic on their health \\ Percepción de los ancianos sobre las repercusiones de la pandemia COVID-19 en su salud
}

Recebido: 09/07/2021 | Revisado: 17/07/2021 | Aceito: 21/07/2021 | Publicado: 29/07/2021

\author{
Elisabelle Martins Marrocos \\ ORCID: https://orcid.org/0000-0001-7967-2317 \\ Centro Universitário Christus, Brasil \\ E-mail: elisabellmarrocos@gmail.com \\ Alisson Salatiek Ferreira de Freitas \\ ORCID: https://orcid.org/0000-0002-4547-5785 \\ Centro Universitário Christus, Brasil \\ E-mail: salatiek@gmail.com \\ Gerarda Maria Carneiro \\ ORCID: https://orcid.org/0000-0003-0254-6173 \\ Centro Universitário Christus, Brasil \\ E-mail: gerarda_gg@hotmail.com \\ Mardenia Gomes Vasconcelos Pitombeira \\ ORCID: https://orcid.org/0000-0003-2969-6526 \\ Centro Universitário Christus, Brasil \\ E-mail: mardeniagomes@yahoo.com.br
}

\begin{abstract}
Resumo
A pandemia por COVID-19 indicam maior taxa de mortalidade entre os idosos. O estudo objetivou caracterizar a percepção dos idosos com relação a influência da pandemia na sua própria saúde. Trata-se de uma pesquisa de levantamento com abordagem quantitativa que utilizou como instrumento de coleta de dados o formulário da plataforma Google Forms de março à maio de 2021, participaram 123 idosos do Brasil. Para a análise utilizou-se a estatística descritiva, calculando as frequências absolutas e relativas. A pesquisa seguiu os critérios éticos da Resolução $n^{\circ}$ 510/2016. Os resultados descrevem a participação de idosos com idade média de 69 anos, sexo feminino entre outros dados sociodemográficos. No impacto da pandemia na saúde em geral, $74 \%$ informaram não terem sido acometidos por COVID-19, os que precisaram ser internados, a maioria tinham comorbidades como diabetes melitus e hipertensão arterial sistêmica. Os que não tiveram COVID-19, 26\% deles informaram que adicionaram medicamentos na sua rotina. Referente as mudanças na rotina por conta da pandemia a percepção deles é de que a pandemia por COVID-19 mudou pouca coisa, consideram não terem ficado mais dependentes, mas que houve uma mudança na rotina com o isolamento social, inclusive com os cuidados de saúde. Um tema relevante foi a prevalência de idosos que expressam o sentimento de medo, especialmente o medo de perder um ente querido. Apesar de tudo esse período possibilitou novos aprendizados. Conclui-se que as influências da pandemia por COVID-19 estão relacionadas principalmente a sentimentos de medo, isolamento social, automedicação, mas também de aprendizado.
\end{abstract}

Palavras-chave: Idoso; Pandemias; Saúde; Automedicação; Mídias sociais.

\begin{abstract}
The COVID-19 pandemic indicates a higher mortality rate among the elderly. The study aimed to characterize the perception of the elderly regarding the influence of the pandemic on their own health. This is a survey with a quantitative approach that used the form of the Google Forms platform as a data collection instrument from March to May 2021. 123 elderly people from Brazil participated. For the analysis, descriptive statistics were used, calculating the absolute and relative frequencies. The research followed the ethical criteria of Resolution No. 510/2016. The results describe the participation of elderly people with a mean age of 69 years, female, among other sociodemographic data. Regarding the impact of the pandemic on health in general, $74 \%$ reported not having been affected by COVID-19, those who had to be hospitalized, most had comorbidities such as diabetes mellitus and systemic arterial hypertension. Of those who did not have COVID-19, 26\% of them reported that they added medication to their routine. Regarding the changes in routine due to the pandemic, their perception is that the COVID19 pandemic has changed little, they consider that they have not become more dependent, but that there was a change in the routine with social isolation, including health care. A relevant theme was the prevalence of elderly people expressing the feeling of fear, especially the fear of losing a loved one. Despite everything, this period allowed for
\end{abstract}


new learning. It is concluded that the influences of the COVID-19 pandemic are mainly related to feelings of fear, social isolation, self-medication, but also to learning.

Keywords: Elderly; Pandemics; Health; Self-medication; Social media.

\begin{abstract}
Resumen
La pandemia de COVID-19 indica una mayor tasa de mortalidad entre los ancianos. El estudio tuvo como objetivo caracterizar la percepción de los ancianos sobre la influencia de la pandemia en su propia salud. Se trata de una encuesta con enfoque cuantitativo que utilizó el formulario de la plataforma Google Forms como instrumento de recolección de datos de marzo a mayo de 2021. Participaron 123 personas mayores de Brasil. Para el análisis se utilizó estadística descriptiva, calculando las frecuencias absolutas y relativas. La investigación siguió los criterios éticos de la Resolución No. 510/2016. Los resultados describen la participación de personas mayores con una edad media de 69 años, mujeres, entre otros datos sociodemográficos. En cuanto al impacto de la pandemia en la salud en general, el $74 \%$ refirió no haber sido afectado por COVID-19, los que tuvieron que ser hospitalizados, la mayoría presentaba comorbilidades como diabetes mellitus e hipertensión arterial sistémica. De los que no tenían COVID-19, el 26\% de ellos informaron que agregaron medicamentos a su rutina. En cuanto a los cambios en la rutina por la pandemia, su percepción es que la pandemia COVID-19 ha cambiado poco, consideran que no se han vuelto más dependientes, pero que hubo un cambio en la rutina con aislamiento social, incluido el cuidado de la salud. Un tema relevante fue la prevalencia de personas mayores que expresaron el sentimiento de miedo, especialmente el miedo a perder a un ser querido. A pesar de todo, este período permitió nuevos aprendizajes. Se concluye que las influencias de la pandemia COVID-19 se relacionan principalmente con sentimientos de miedo, aislamiento social, automedicación, pero también con el aprendizaje.
\end{abstract}

Palabras clave: Anciano; Pandemias; Salud; Automedicación; Redes sociales.

\title{
1. Introdução
}

O mundo está enfrentando a pandemia por COVID-19 causada por um vírus conhecido como novo coronavírus (SarsCov-2). No Brasil, o primeiro caso positivo foi anunciado em 26 de fevereiro de 2020, sendo um homem morador de São Paulo, com 61 anos, que esteve na Itália. O primeiro óbito, oficialmente relacionado ao COVID-19, no Brasil ocorreu em 17 de março de 2020, de um homem, 62 anos, diabético e hipertenso. Os dados do COVID-19 indicam maior taxa de mortalidade entre as pessoas com 80 anos ou mais, trazendo os idosos para o centro da discussão da pandemia COVID-19 e exigindo uma atenção especial tanto na prevenção quanto no tratamento (Hammerschmidt, 2020).

Segundo a Organização Mundial de Saúde (OMS) é considerado idoso qualquer pessoa com idade acima de 60 anos, lembrando que essa categorização foi feita exclusivamente segundo o seu envelhecimento físiológico, o que não impede de ser um indivíduo social e intelectualmente ativo (Monteiro \& Coutinho, 2020).

No mundo, com o avanço tecnológico, o aumento da escolaridade, da renda e a melhoria de outros fatores determinantes, as taxas de natalidade e de mortalidade estão reduzindo e a expectativa de vida aumentando, elevando proporcionalmente o número de pessoas com 60 anos ou mais, a isso chamamos de transição demográfica (Nascimento \& Diógenes, 2020).

O Brasil tem mais de 28 milhões de pessoas nessa faixa etária, representando $13 \%$ da população, podendo dobrar nas próximas décadas, segundo a Projeção da População, divulgada em 2018 pelo Instituto Brasileiro de Geografia e Estatística IBGE. Dentre as principais causas para essa tendência de envelhecimento, como já citado anteriormente, temos a queda da taxa de fecundidade e o aumento da expectativa de vida do brasileiro (IBGE, 2018).

$\mathrm{Na}$ mesma velocidade do aumento da expectativa de vida, crescem também os desafios para com esse segmento da população, principalmente no quesito proteção e assistência à saúde, o que gera impactos diretos no Sistema Único de Saúde (SUS), pois os idosos são a faixa populacional que mais utiliza o serviço (Machado, 2017).

Diante do exposto, tornou-se evidente a necessidade de pesquisar sobre o estado da saúde dos idosos visto que isso pode contribuir para elaboração de planos de cuidados e atividades em educação em saúde. O presente estudo tem como objetivo caracterizar a percepção das pessoas com 60 anos ou mais com relação a influência da pandemia por COVID-19 na 
sua própria saúde.

\section{Metodologia}

Trata-se de uma pesquisa do tipo levantamento, com abordagem quantitativa que busca mapear as características de uma determinada população diante da pandemia por COVID-19 realizada durante o primeiro semestre de 2021. As pesquisas do tipo levantamento se caracterizam pela interrogação direta do grupo cujo comportamento se deseja conhecer, após a solicitação de informações a uma quantidade significativa de pessoas é feita a análise quantitativa e procede com o estudo resultando das conclusões referente aos dados coletados (Gil, 2017).

Os participantes do estudo foram 123 idosos, como critério de inclusão foram definidos: pessoas com 60 anos ou mais, com acesso a internet e que sabiam utilizar a tecnologia ou tivessem alguém para o ajudar. Foram excluídos os formulários incompletos ou enviados fora do período de coleta de dados.

A coleta de dados ocorreu durante o mês de março a maio de 2021, por meio do compartilhamento do link do formulário online para idosos de todo o Brasil. A consulta foi feita por meio de um questionário elaborado virtualmente na plataforma Google Forms contendo perguntas sobre o perfil sociodemográfico, estado de saúde e outras mudanças pós pandemia de COVID-19, tendo a participação de pessoas de forma espontânea e totalmente voluntária.

A captação ou o convite para os participantes na pesquisa, ocorreu pelo método bolo de neve (snowball) que é uma técnica de amostragem que vem sendo utilizada em pesquisas nos últimos anos, principalmente por ser bastante útil para pesquisar grupos difíceis de serem estudados ou acessados ou também quando não se conhece o universo da pesquisa. A técnica de bola de neve pode ser definida como uma técnica de amostragem que utiliza de cadeias de referência, como uma rede para coleta de informações, usando técnicas de recrutamento em cadeia (Bockorni \& Gomes, 2021).

Diante deste cenário pandêmico se fez necessário utilizar novas maneiras de fazer pesquisas mantendo a distância imposta pela reclusão e afastamento social e uma delas foi a ferramenta Google Forms, apoiando os discentes e docentes em seus trabalhos acadêmicos (Martins, 2020).

O Google Forms é um aplicativo que pode criar formulários, esses podem ser questionários de pesquisa, que ficam armazenados no Google Drive. É um serviço gratuito, exigindo apenas uma conta no Gmail e por ficarem armazenados no Servidor do Google, podem ser acessados de qualquer lugar e não ocupam espaço no computador. A maior vantagem da utilização dessa ferramenta para a pesquisa, é que o autor pode enviar para os participantes via E-mail ou WhatsApp, pois o mesmo gera um link onde quem tiver de posse do mesmo, poderá responder de onde estiver (Mota, 2019).

Utilizou-se a análise estatística com frequências absolutas e relativas através do IBM SPSS Statistics e recursos do próprio excel, os resultados foram apresentados em gráficos e tabelas com o auxílio do excel e powerpoint. Os dados foram interpretados e discutidos com a literatura pertinente ao tema e o referencial teórico sustentadas pelos autores citados.

A pesquisa seguiu os preceitos éticos descritos na Resolução n ${ }^{\circ} 510$ do Conselho Nacional de Saúde (CNS), promulgada em 2016. Ela determina procedimentos éticos específicos para investigações com seres humanos, que utilizam abordagens das Ciências Humanas e Sociais (CHS). Destaca ainda em seu Artigo $1^{\circ}$, Parágrafo Único que a referida resolução indica que pesquisas de opinião pública com participantes não identificados não necessitam ser enviadas ao Comitê de Ética em Pesquisa com Seres Humanos.

Essa pesquisa faz parte de uma pesquisa mais ampla, denominada "Itinerários Terapêuticos de Idosos com Depressão", no qual foi aprovado pelo comitê de ética em pesquisa da Unichristus com o parecer 2.198.647 do ano de 2017. 


\section{Resultados e Discussão}

As características sociodemográficas dos idosos que responderam a pesquisa estão expressas na Tabela 1. Nota-se que 65\% dos idosos são do sexo feminino. A faixa etária foi dividida em três grupos, onde prevaleceu, com 65\%, as idades entre 60 e 70 anos, $28 \%$ as idades de 71 a 80 anos e com apenas $7 \%$ pessoas com idade igual ou superior a 80 anos, a média de idade da pesquisa foi de 69 anos. Como escreveu Alves (2019), “o futuro do século XXI será grisalho", ou seja, o percentual mundial de idosos alcançará cifras recordes, sendo assim as economias mundiais sendo assim as economias mundiais precisam se adaptar, preparando-se para lidar com uma estrutura etária culturalmente afastada das atividades produtivas, além das consequências, para a Rede de Atenção à Saúde, de uma alta razão de dependência demográfica.

O nível de escolaridade dos entrevistados ficou com 41\% de "Ensino superior completo", 23\% "Primeiro grau completo" e em terceiro lugar com 15\% o "Segundo grau completo" o que pode ser explicado pelo uso de uma consulta online, onde, para responder, o idoso precisa não só ter acesso a tecnologia, como ter conhecimento para saber usá-la.

Neste contexto, reforçasse-se a importância da inclusão tecnológica para a população idosa, visto que é uma tendência cada vez mais presente a tecnologia no dia a dia da sociedade, o que foi intensificado pela pandemia da COVID-19.

Com relação ao estado civil, nota-se que a maior porcentagem é de casados, com $41 \%$ e em segundo lugar, com 23\%, os viúvos. Quanto a localidade dos idosos que responderam a maior porcentagem foi do Ceará (Nordeste) com 62\%. Uma parcela significativa, que correspondem a 70\%, responderam que são aposentados ou pensionistas.

Ao analisar as comorbidades dos entrevistados, $27 \%$ responderam que não tem nenhuma comorbidades, $1 \%$ possui apenas diabetes melitus - DM, 12\% apenas hipertensão arterial sistêmica - HAS, 2\% apenas com cardiopatias, $2 \%$ com artrite, 4\% com artrose, $6 \%$ com outros (Não identificados) e 6\% possuem DM e HAS juntos. Ainda sobre as comorbidades pode-se analisar que $12 \%$ possuem duas comorbidades associadas além da HS e DM já mencionada anteriormente, e $29 \%$ referiram mais de duas comorbidades associadas, como por exemplo, Diabetes, HAS, Cardiopatias, Dislipidemia, Asma e outras Doenças Pulmonares, Artrite, Artrose, Obesidade, Reumatismo, Doenças renais e outras. 
Research, Society and Development, v. 10, n. 9, e41010918067, 2021

(CC BY 4.0) | ISSN 2525-3409 | DOI: http://dx.doi.org/10.33448/rsd-v10i9.18067

Tabela 1 - Características sociodemográficos dos idosos pesquisados. Fortaleza, Ceará, Brasil.

\begin{tabular}{|c|c|c|c|}
\hline Variáveis & & $\mathrm{N}$ & $\%$ \\
\hline \multirow[t]{2}{*}{ Sexo } & Feminino & 80 & $65 \%$ \\
\hline & Masculino & 43 & $35 \%$ \\
\hline \multirow[t]{4}{*}{ Idade } & 60 a 70 anos & 80 & $65 \%$ \\
\hline & 71 a 80 anos & 34 & $28 \%$ \\
\hline & Mais de 80 anos & 9 & $7 \%$ \\
\hline & Média & 69 & $56 \%$ \\
\hline \multirow[t]{7}{*}{ Escolaridade } & Primeiro grau incompleto & 28 & $23 \%$ \\
\hline & Primeiro grau completo & 5 & $4 \%$ \\
\hline & Segundo grau incompleto & 7 & $6 \%$ \\
\hline & Segundo grau completo & 18 & $15 \%$ \\
\hline & Ensino superior incompleto & 5 & $4 \%$ \\
\hline & Ensino superior completo & 51 & $41 \%$ \\
\hline & Não se aplica a nenhuma das opções & 9 & $7 \%$ \\
\hline \multirow[t]{5}{*}{ Situação Conjugal } & Casado(a) & 51 & $41 \%$ \\
\hline & Viúvo(a) & 28 & $23 \%$ \\
\hline & Solteiro(a) & 19 & $15 \%$ \\
\hline & Outro & 17 & $14 \%$ \\
\hline & União estável & 8 & $7 \%$ \\
\hline \multirow[t]{8}{*}{ Localidade } & Ceará - Nordeste - Brasil & 76 & $62 \%$ \\
\hline & São Paulo - Sudeste - Brasil & 4 & $3 \%$ \\
\hline & Rio de Janeiro - Sudeste - Brasil & 3 & $2 \%$ \\
\hline & Tocantins - Norte - Brasil & 3 & $2 \%$ \\
\hline & Paraná - Sul - Brasil & 2 & $2 \%$ \\
\hline & Goiás - Centro-Oeste - Brasil & 1 & $1 \%$ \\
\hline & Atlanta & 1 & $1 \%$ \\
\hline & Não informaram & 33 & $27 \%$ \\
\hline \multirow[t]{2}{*}{ Aposentado/Pensionista } & Sim & 86 & $70 \%$ \\
\hline & Não & 37 & $30 \%$ \\
\hline \multirow[t]{10}{*}{ Comorbidades } & Sem comorbidades & 33 & $27 \%$ \\
\hline & Apenas Diabetes (DM) & 1 & $1 \%$ \\
\hline & Apenas Hipertensão (HAS) & 15 & $12 \%$ \\
\hline & Apenas Cardiopatias & 2 & $2 \%$ \\
\hline & Apenas Artrite & 2 & $2 \%$ \\
\hline & Apenas Artrose & 5 & $4 \%$ \\
\hline & Apenas Outros & 7 & $6 \%$ \\
\hline & Apenas DM e HAS & 7 & $6 \%$ \\
\hline & Outras duas comorbidades associadas & 15 & $12 \%$ \\
\hline & Mais de duas comorbidades associadas & 36 & $29 \%$ \\
\hline
\end{tabular}

Fonte: Autores.

Sabe-se que a hipertensão e diabetes são doenças que andam juntas na maioria das vezes, visto que, a diabetes causa alterações vasculares e o alto índice dessas duas comorbidades na pessoa idosa, carreta em fatores de envelhecimento no sistema cardiovascular e órgãos secretores do hormônio insulina. A HAS é a doença crônica mais prevalente em idosos, porém assim como a DM pode ser controlada (Ribeiro et al., 2020).

Considerando apenas os idosos com 80 anos ou mais e com relação as comorbidades, o resultado que está apresentado na Figura 1 mostra 6\% refere não ter nenhuma comorbidades, 31\% apenas uma comorbidades, 50\% possuem duas comorbidades associadas e 13\% possuem mais de duas comorbidades. 
Figura 1 - Comorbidades dos idosos com 80 anos ou mais. Fortaleza, Ceará, Brasil.

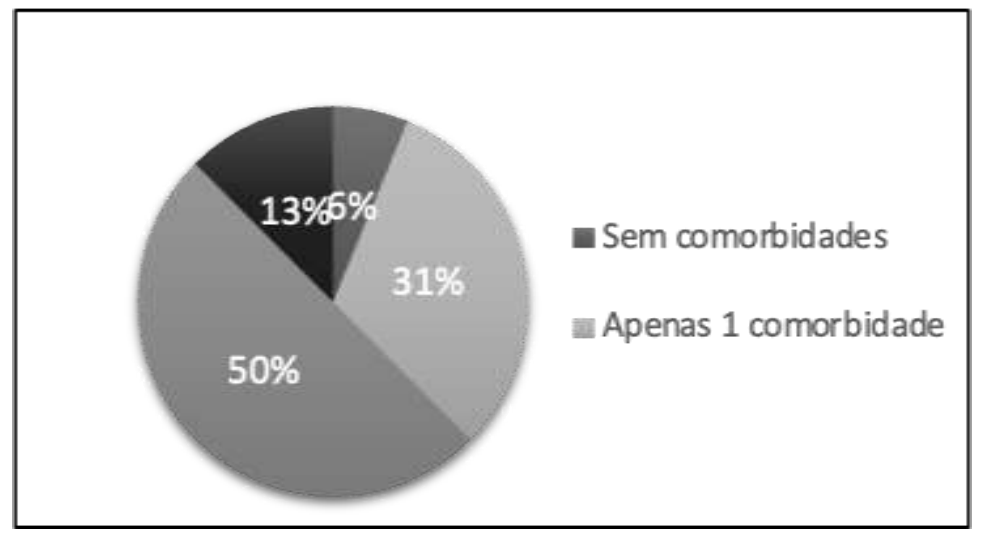

Fonte: Autores.

Estudos mostram que indivíduos de todas idades podem ser infectados pelo novo coronavírus, contudo as manifestações e a gravidade da doença demonstram ser mais exacerbadas de acordo com o aumento da idade e presença de comorbidades, sendo assim os idosos são o principal grupo de risco da COVID-19 (Ferreira, Lasmar \& Ramos, 2021).

Para analisar os dados coletados frente ao objetivo do estudo, apresenta-se duas temáticas para apresentação e discussão dos resultados.

\subsection{Impacto da pandemia na saúde em geral da pessoa idosa}

Sobre a pergunta aos idosos se foram acometidos por COVID-19, 74\% informaram que "não", 16\% disseram que "sim", foram diagnosticados com vírus, mas tiveram sintomas leves, 7\% responderam que não fizeram o teste, mas tiveram sintomas e apenas $2 \%$ ficaram internados para tratar o COVID-19, mas não precisaram de intubação orotraqueal.

Dos idosos que tiveram COVID-19 com sintomas leves, a maior prevalência das doenças crônicas não transmissíveis - DCNT, foi de HAS e DM, como mostra a Figura 2.

Figura 2 - Distribuição das Comorbidades em idosos que tiveram COVID-19 com sintomas leves. Fortaleza, Ceará, Brasil.

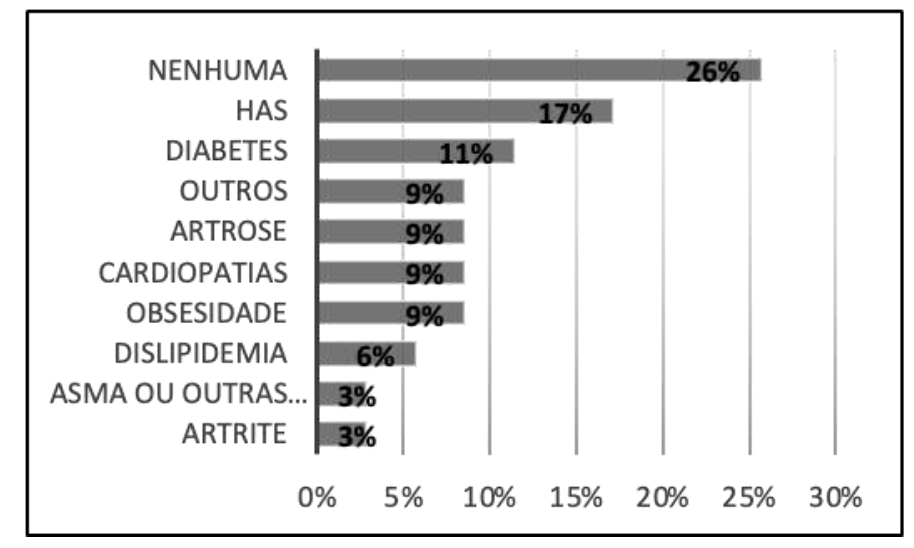

Fonte: Autores.

Sabe-se que a Diabetes e Hipertensão representam um problema de saúde pública, com alto impacto sobre o Sistema 
Único de Saúde - SUS, no contexto da emergência de saúde pública global provocada pela pandemia por COVID-19 torna-se ainda mais relevante a discussão sobre os múltiplos aspectos que envolvem tais doenças, principalmente quando lembrando que a primeira morte pelo novo coronavírus confirmada no Brasil, no estado de São Paulo foi de um homem de 62 anos com hipertensão e diabetes (Almeida \& Neto,2021).

Apesar dos idosos que foram diagnosticados com COVID-19 e ficaram internados, eles referiram que não precisaram adicionar medicamentos a sua rotina e dos que foram diagnosticados com COVID-19 (sintomas leves) 65\% deles referiram que também não precisaram adicionar medicamentos na sua rotina, sendo assim os $35 \%$ restante informaram que adicionaram medicamentos listados na Tabela 2.

Tabela 2 - Medicamentos citados pelos idosos que tiveram COVID-19 com sintomas leves. Fortaleza, Ceará, Brasil.

\begin{tabular}{ll}
\hline Medicamentos & \\
\hline & Dorflex \\
Ivermectina \\
Azitromicina \\
Vitaminas em \\
geral \\
Betabloqueador \\
Zinco \\
Hidroxicloroquina \\
Nimesulida \\
Antitermico \\
Anticoagulante \\
\hline
\end{tabular}

Fonte: Autores.

No dia 18 de maio de 2020, a Associação de Medicina Intensiva Brasileira, a Sociedade Brasileira de Infectologia e a Sociedade Brasileira de Pneumologia e Tisiologia publicam, na Revista da Associação Brasileira de Medicina Intensiva, um artigo de consenso sobre o tratamento da COVID-19 em que recomendam a não utilização da "hidroxicloroquina ou cloroquina de rotina no tratamento da COVID-19", avaliando o grau de indicação como fraco, dado o nível baixo de evidência em favor dessa conduta (Dall'agnol, Azevedo \& Bonella, 2020). Estudos mostram que o uso descontrolado da Hidroxicloroquina tem como principais reações adversas a retinopatia e a arritmia em pacientes com doenças reumatoides (Touret \& Lamballerie, 2020).

Com relação aos que não tiveram COVID-19, 26\% deles informaram que adicionaram medicamentos na sua rotina, os mesmos estão listados na Tabela 3. Segundo Domingues et al. (2017), a automedicação é a seleção e a utilização de medicamentos com intuito de atuar sobre os sintomas das doenças, sem o acompanhamento prévio do profissional de saúde habilitado.

A automedicação - ATM nos idosos apresentam maiores riscos, pois nessa faixa etária apresenta um maior risco de as interações medicamentosas, os aumentos de reações adversas aos medicamentos podem causar complicações aos pacientes de maneira que ele não pode se recuperar devido às alterações típicas do processo de envelhecimento (Silva Filho et al.,2020). 
Tabela 3 - Medicamentos citados pelos idosos que não tiveram COVID-19. Fortaleza, Ceará, Brasil.

\begin{tabular}{ll}
\hline Medicamentos & \\
\hline & Um vermifugo (Não \\
lembra onome) \\
Vitamina C \\
Azitromicina \\
Vitamina D \\
Ivermectina \\
Reconter \\
Zinco \\
Hidroxicloroquina \\
\hline
\end{tabular}

Fonte: Autores.

Estudos internacionais mostram que diante dessa crise pandêmica e da variedade de informações que circulam na internet, plantas e muitas outras substâncias sem os requisitos mínimos de eficácia e têm sido propostas para tratar ou prevenir COVID-19 e esse uso sem orientação médica é considerado automedicação, por conta disso, casos de envenenamento e morte foram relatados nos EUA e na Nigéria em pessoas que se automedicavam com cloroquina o que torna imprescindível intensificar a educação em saúde com campanhas de conscientização para combater a desinformação sobre os produtos de prevenção do COVID-19 nas mídias sociais (Sadio et al., 2021).

Além disso, uma pesquisa online feita para determinar as práticas padrão de automedicação, risco e índice de gravidade da medicação da população em confinamento do Equador através dos critérios do estadiamento do escore de frequência da automedicação por gravidade - HRAS. O resultado mostrou que 55\% da população equatoriana tinha um risco extremo de automedicação com pontuação HRAS - 25 pontos, 35\% preferem uma recomendação de terapia medicamentosa de TV, redes sociais e influenciador em vez de um profissional de saúde com pontuação HRAS - 20 pontos, e 39\% terminam sua medicação antes das recomendações (médico ou farmacêutico), com pontuação de HRAS - 12 ponto o que só evidencia o alto risco da população equatoriana à automedicação, independentemente do nível de escolaridade adquirido e a influência das mídias sociais nessa conduta (Manzano et al., 2021).

Com relação a pergunta como os idosos classificaram sua dependência por ajuda de outras pessoas por conta da pandemia, $45 \%$ informaram que não ficaram dependentes, $24 \%$ informaram que ficaram "um pouco" e $31 \%$ responderam que sim, ficaram dependentes de outras pessoas por causa da pandemia. Estudos mostram que o isolamento social intensificou a perda de autonomia em idosos o que vai contra ao que a própria Organização Mundial de Saúde - OMS prega que, para existir um envelhecimento ativo, é fundamental garantir a independência e a autonomia dos idosos bem como a expectativa de vida saudável e de qualidade (Velho \& Herédia, 2020).

O cuidado gerontológico ganhou destaque devido a vulnerabilidade dos idosos e junto a isso a importância de criar ou resgatar os vínculos com essa população, pois a autonomia e independência do idoso são o alicerce para o envelhecimento saudável, assim, torna-se um desafio trabalhar esse apontamento diante do distanciamento social necessário para preservar a saúde e proteção dessa população (Lima \& Chicone, 2021). 


\subsection{Mudanças na rotina do idoso por conta da pandemia por COVID-19}

Observa-se que, a mudança na rotina quanto a ir tomar vacina da gripe e fazer as consultas médicas anuais, as respostas foram que $56 \%$ tomaram a vacina contra a gripe e $49 \%$ continuaram suas rotinas de exames e médicos para manter a saúde em dia, ou seja, aproximadamente metade dessa população continuou com esses cuidados e a outra metade não, o que pode causar impacto no controle e prevenção de agravos nas DCNT e outra doenças adquiridas durante a pandemia. Durante esse período de pandemia em que os idosos devem permanecer em isolamento social, nota-se que, no Brasil, esse isolamento não é tão satisfatório assim para garantir as medidas de segurança (Oliveira, Sousa \& Rocha Filho (2021).

Com relação aos sentimentos que esses idosos começaram a ter ou ter com mais frequência após a pandemia o que teve maior prevalência foi o "Medo de perder um ente querido" com $70 \%$, em segundo lugar foi "Medo de ficar internado" com 46\% das respostas, 40\% informaram "Tristeza" e "Ansiedade", 33\% "Medo de morrer", 24\% "Solidão", $10 \%$ sentiram "Abandono" e por fim 6\% estão com medo de ficar desempregado, conforme a Figura 3, lembrando que a soma dos percentuais não supera $100 \%$ porque alguns entrevistados responderam mais de um sentimento, uma vez que a pergunta era de múltipla escolha.

Figura 3 - Sentimentos após a pandemia por COVID-19. Fortaleza, Ceará, Brasil.

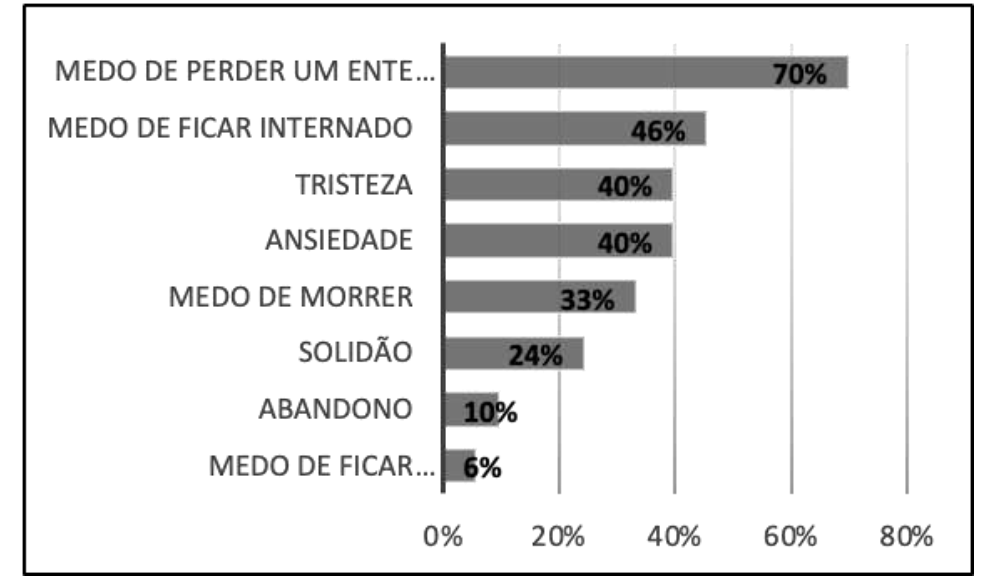

Fonte: Autores.

Estudos internacionais afirmam que os idosos são mais suscetíveis aos efeitos psicológicos em uma pandemia, sendo assim eles podem mais facilmente manifestar emoções como tristeza, ansiedade, estresse, raiva, medo e choro. Além disso, idosos com comorbidades possuírem duas vezes maior chance de ter chorado muitas vezes durante a pandemia, quando comparado aos sem comorbidades, se explica pela incerteza da cura e/ou da morte do idoso caso seja infectado (Santini et al., 2020; Banerjee,2020). O choro, por sinal, é uma das manifestações de transtornos mentais, como pode ser observado em um estudo realizado com idosos chineses, relatando depressão e ansiedade durante a pandemia da COVID-19 (Souza et al.,2021).

Nesse sentido, enfatiza-se a preocupação com um possível aumento de casos/sintomas depressivos em idosos, durante e após o início da pandemia por COVID-19, por conta da relação dessa doença com casos de demência, o que pode elevar o número de idosos dependentes totais no futuro, sobrecarregando serviços de saúde e a sociedade por meio dos familiares e cuidadores.

A pandemia por COVID-19, além de expor as fragilidades e particularidades da população idosa, trouxe diversos impactos na vida social, alterações físicas, mentais e psicossociais, por isso é importante que cuidadores e familiares fiquem atentos aos primeiros sinais de tristeza, desânimo e falta de energia (Oliveira, Sousa \& Rocha Filho, 2021). 
A baixa procura dos serviços de saúde é um dos principais agravos causados pelo distanciamento social o que retarda esse contato para um momento mais crítico, já que ambientes hospitalares e ambulatoriais são locais de grande movimentação de pessoas que provavelmente estão com alguma enfermidade, podendo, inclusive, estarem contaminadas pelo coronavírus. Para diminuir tanto a demanda nesses lugares quanto para garantir maior segurança da população de risco, as instituições de saúde estão promovendo atendimentos on-line (Vidal \& Castro, 2020).

Referente a pergunta sobre o aumento do contato com a tecnologia, nota-se que a pandemia trouxe esse impacto positivo na vida dessa população, pois $61 \%$ dos entrevistados responderam que tiveram esse contato ou contato maior com a tecnologia, enquanto apenas $18 \%$ relataram que já usavam bem antes da pandemia. Contudo, 21\% dos idosos informaram que não gostam de tecnologia e não tiveram esse contato maior conforme mostra a Figura 4.

Estudos apontam que o acesso à tecnologia, ou seja, a chamada alfabetização digital tem demonstrado ser um elemento-chave na capacidade de lidar com os desafios impostos pela quarentena (Silva et al., 2021).

O objetivo principal do uso de tecnologias é diminuir a influência negativa das restrições dos idosos na participação em atividades rotineiras, além de ser um meio de promover conexões sociais (Seifert, Cotten \& Xie, 2021).

Figura 4 - Aumento do contato com a Tecnologia (Smartphone, Notebook, Internet e etc). Fortaleza, Ceará, Brasil.

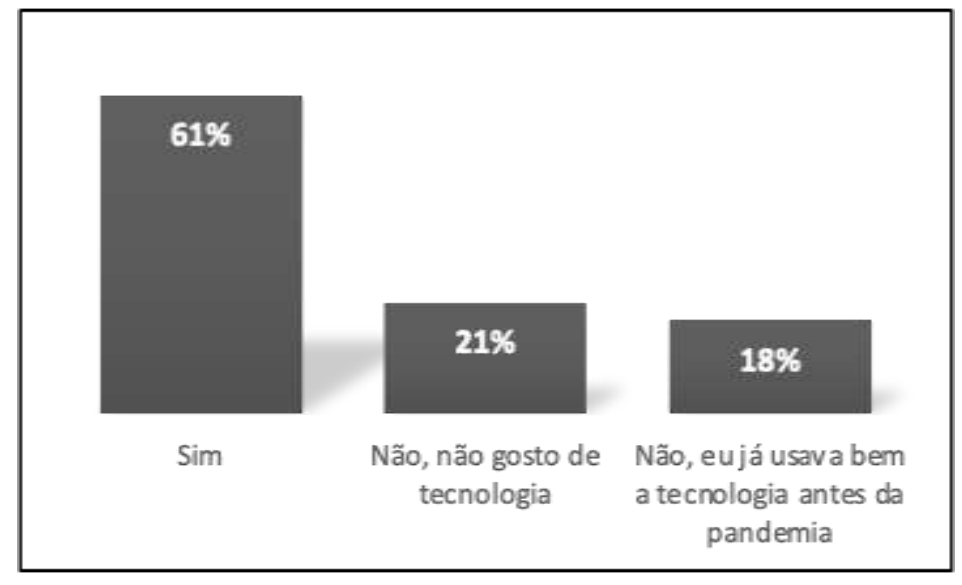

Fonte: Autores.

O desenvolvimento e a disseminação da internet em todo o mundo têm contribuído para aumentar as preocupações entre pesquisadores e profissionais de saúde sobre o uso problemático da Internet - PIU, levando a Organização Mundial da Saúde (OMS) a examinar, desde 2014, suas implicações para a saúde pública. Um estudo internacional mostrou que embora os idosos façam parte da população com o nível mais baixo de utilização da Internet, o acesso e a utilização da Internet entre eles aumentaram e está aumentando progressivamente na Europa e em todo o mundo. Isso ocorre não somente por causa da cobertura de marketing, mas também porque os atuais jovens adultos que usam a internet serão os usuários mais velhos no futuro. Além disso esse foi o primeiro estudo a examinar o uso excessivo da Internet em uma amostra de idosos e o mesmo traz uma necessidade de levar em consideração antecedentes culturais e sociodemográficos ao examinar o uso e mau uso da Internet, pois a PIU pode estar presente em um subgrupo de usuários idosos da Internet e que a Internet pode ser usada para lidar com emoções negativas ou insatisfação com a vida e por isso é necessário promover estratégias eficazes de prevenção e tratamento personalizado (Rochat et al., 2021).

$\mathrm{Na}$ pergunta sobre novos aprendizados durante a pandemia, 45\% dos idosos responderam que "Sim", aprenderam coisas novas e desses as falas que mais se destacaram foram "Tô fazendo faculdade", "Ler mais livros", "Fazer atividade física em casa", "Economizar" entre outras que estão elas estão expressa na Tabela 4. 
Tabela 4 - Lista de novos aprendizados. Fortaleza, Ceará, Brasil.

\begin{tabular}{|c|c|}
\hline Aprendizados & \\
\hline & Tô fazendo faculdade \\
\hline & $\begin{array}{l}\text { Fiz um curso de auxiliar na área de } \\
\text { qualidade }\end{array}$ \\
\hline & Fazer atividade fisica em casa \\
\hline & Voltei a caminhar e a fazer crochê. \\
\hline & Aprendi a fazer mascara \\
\hline & Estudar espanhol \\
\hline & Cuidar da cozinha e fazer as refeições \\
\hline & Ler mais livros \\
\hline & Fazer produtos de limpeza pela internet \\
\hline & Economizar \\
\hline
\end{tabular}

Fonte: Autores.

Ao pedir para os participantes da pesquisa marcarem as opções que indicavam mudanças na sua rotina após a pandemia por COVID-19, nota-se que 67\% não comparecem mais aos encontros dos amigos, 63\% não vão mais às festas da família, $60 \%$ não vão ao centro da cidade mais, $53 \%$ não vão ao shopping, $48 \%$ não vão mais para a academia/fisioterapia, $42 \%$ deixaram de ir ao banco, $30 \%$ deixaram de fazer acompanhamento médico, $18 \%$ deixaram de receber visitas dos filhos e netos e apenas $13 \%$ informaram que sua rotina continua a mesma de antes da pandemia conforme mostra a Figura 5.

Figura 5 - Mudança na rotina do idoso após a pandemia por COVID-19. Fortaleza, Ceará, Brasil.

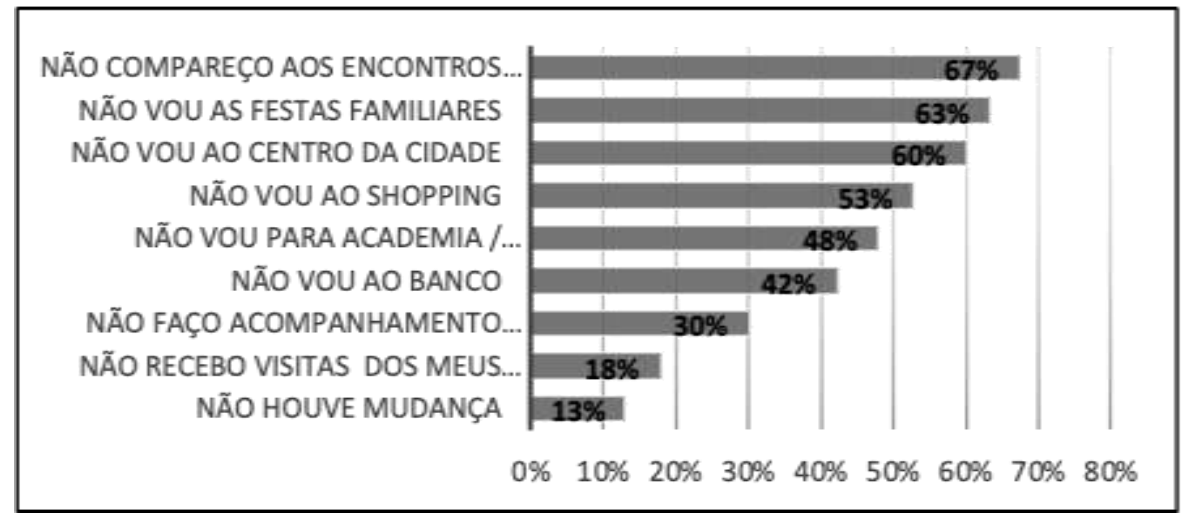

Fonte: Autores.

O comportamento social na velhice, muitas vezes é caracterizado pela redução das redes sociais e diminuição da participação em atividades coletivas, além do acesso ou a capacidade limitada de usar as tecnologias digitais, o que pode impedi-los ou dificultar a obtenção de bens, serviços e apoio social necessários durante esse contexto pandêmico. (Silva et al., 2021).

Apesar dos idosos fazerem parte do grupo de risco, é preciso compreender que a velhice, em seu processo natural, apresenta inúmeras peculiaridades, e que a rotina dos idosos precisa ser mantida (Quintino, 2021). Manter a capacidade funcional do idoso significa preservar as habilidades físicas e mentais necessárias para uma vida independente e autônoma, pois sabe-se que em pessoas idosas as doenças crônicas diante da inatividade ficam mais acentuadas, o que pode agravar e impedir de realizar as Atividades de Vida Diária - AVD, limitando sua interação e convívio social, situações estas que 
diminuem consideravelmente sua qualidade de vida. (Medeiros et al., 2021).

\section{Conclusão}

Observou-se que a pandemia por COVID-19 influenciou sentimentos, atitudes e rotina da população idosa. Entre os sentimentos compartilhados pelos idosos, o medo de perder uma pessoa querida foi o mais prevalente. Esta emoção ganha mais relevância na população idosa, em virtude desse grupo apresentar mais riscos de adquirir a forma grave da doença, bem como de vivenciaram perdas de pessoas da mesma faixa etária.

Um comportamento que foi evidenciado pela pesquisa foi a automedicação dos idosos. De acordo com os participantes a atitude de acrescentar medicamentos, como vitaminas, antiparasitários, antibióticos, entre outros, foi mais prevalente neste período e que deve ser avaliado pelo profissional de saúde, uma vez que a maioria dos idosos já faz uso de outros medicamentos, com o risco de efeitos indesejados na saúde do idoso pela polifarmácia.

Além desses, a rotina dos idosos foi impactada pela medida de isolamento social e distanciamento físico, que impediu idosos de exercitarem a autonomia especialmente de exercitarem a sua vida social com amigos e familiares. Fato este que pode trazer consequências para a saúde mental do idoso. Por outro lado, esse período possibilitou o aprendizado de outras habilidades como a utilização de tecnologia e desenvolvimento intelectual para alguns idosos.

O crescimento progressivo do número de usuários idosos na Internet torna necessário mais pesquisas para avaliar melhor os fundamentos psicológicos, biológicos, sociais e culturais do uso problemático da internet nesse público e promover estratégias para a prevenção e tratamento individualizado de forma eficiente.

Como limitação do estudo, tem-se a forma de coleta de dados utilizada, que exclui idosos de classes sociais mais baixas, que não tem acesso a internet e nem sabe utilizar o smartphone para responder o questionário de pesquisa. Desse modo, recomenda-se a realização de futuras pesquisas que possam abranger uma diversidade de idosos para conhecer outras repercussões da pandemia por COVID-19 na saúde do idoso.

\section{Referências}

Almeida, T. A., \& Neto, M. D. C. G. (2021). O HiperDia no contexto da pandemia da COVID-19. Journal of Multiprofessional Health Research, 2(1), e02-47.

Alves, J. E. D. (2019). Envelhecimento populacional no Brasil e no mundo. Revista Longeviver.

Banerjee, D. (2020). 'Age and ageism in COVID-19': Elderly mental health-care vulnerabilities and needs. Asian journal of psychiatry, 51, 102154.

Bockorni, B. R. S., \& Gomes, A. F. (2021). A amostragem em snowball (bola de neve) em uma pesquisa qualitativa no campo da administração. Revista de Ciências Empresariais da UNIPAR, 22(1).

Domingues, P. H. F., Galvão, T. F., Andrade, K. R. C. D., Araújo, P. C., Silva, M. T., \& Pereira, M. G. (2017). Prevalência e fatores associados à automedicação em adultos no Distrito Federal: estudo transversal de base populacional. Epidemiologia e Serviços de Saúde, 26, 319-330.

Silva, M. F., Silva, D. S. M. D., Bacurau, A. G. D. M., Francisco, P. M. S. B., Assumpção, D. D., Neri, A. L., \& Borim, F. S. A. (2021). Ageismo contra idosos no contexto da pandemia da covid-19: uma revisão integrativa. Revista de Saúde Pública, 55, 4.

Nascimento, M. V. D. (2017). Um estudo sobre o impacto do envelhecimento populacional na previdência social.

Da Silva Mota, J. (2019). Utilização do google forms na pesquisa acadêmica. Humanidades \& Inovação, 6(12), 371-373.

De Almeida Hammerschmidt, K. S., \& Santana, R. F. (2020). Saúde do idoso em tempos de pandemia COVID-19. Cogitare enfermagem, 25.

Dall'Agnol, D., de Azevedo, M. A., \& Bonella, A. E. 10. Princípios bioéticos, medicina-baseada-em-evidências e opções terapêuticas no tratamento da covid-191.

Da Paz Silva Filho, P. S., da Costa, R. E. A. R., da Silva Andrade, I. A., dos Santos Sousa, F. W., Júnior, J. D. S. A., Neto, A. S. C., ... \& de Sousa, C. (2020). Riscos da automedicação em idosos acometidos pelo coronavírus e outras síndromes respiratórias. Research, Society and Development, 9(7), e458974211-e458974211. 
De Lima, A. B. L., \& Chicone, M. C. (2021, May). O impacto da pandemia na saúde e vida diária do idoso. In Anais do Congresso de Geriatria e Gerontologia do UNIFACIG (Vol. 1, No. 1)

De Souza Quintino, A. S., de Souza, S. C., Teixeira, P. G., \& Neto, F. E. (2021). O impacto do envelhecimento em tempos de pandemia e o isolamento social na terceira idade. linkscienceplace-Interdisciplinary Scientific Journal, 7(3).

Ferreira, L. B., Lasmar, M. F., \& Ramos, D. F. (2021). Mudanças comportamentais da população da cidade de Rio Grande (RS) frente à pandemia. Research, Society and Development, 10(2), e29010212413-e29010212413.

GIL, A. C. (2017). Como elaborar projetos de pesquisa. 6. ed. São Paulo: Atlas

Brasil, I. B. G. E. Instituto Brasileiro de Geografia e Estatıstica, Censo demográfico 2018.

Manzano, A. O., Manzano, S. O., Sanchez, L. D., Vizcaino, M., Gomez - Franco, F., \& Chuquimarca - Tandazo, L. Risco de auto - medicação durante a pandemia de confinamento SARS - COV - 2. The FASEB Journal, 35

Martins, F. A. (2020, August). Google forms como ferramenta de apoio: experiência docente em meio a pandemia corona vírus. In Anais do CIET: EnPED: 2020-(Congresso Internacional de Educação e Tecnologias| Encontro de Pesquisadores em Educação a Distância).

Meleiro, M. L. D. A. P., Brito, K. M. D. S. M., \& Nascimento, I. R. (2020). Marcos legais e políticas públicas para idosos no Brasil e no Amazonas. Revista Kairós: Gerontologia, 23(3), 277-298.

Monteiro, R. E. G., \& Coutinho, D. J. G. (2020). Uma breve revisão de literatura sobre os idosos, o envelhecimento e saúde. Brazilian Journal of Development, 6(1), 2358-2368.

Moura, M. L. S. D. (2021). Idosos na pandemia, vulnerabilidade e resiliência.

Medeiros, F. D. A. L., Freitas, E. P. S., Medeiros, A. C. T., \& Medeiros, F. A. L. Reflexões sobre o enfrentamento da dor crônica durante a pandemia da covid-19.

Oliveira, AMC, da Silva Sousa, E., e da Rocha Filho, DR (2021). Alterações físicas, emocionais e psicossociais de idoso na pandemia por coronavírus. Research, Society and Development, 10 (6), e44310615964-e44310615964.

Oliveira, AMC, da Silva Sousa, E., e da Rocha Filho, DR (2021). Alterações físicas, emocionais e psicossociais de idoso na pandemia por coronavírus. Research, Society and Development , 10 (6), e44310615964-e44310615964.

Rochat, L., Wilkosc-Debczynska, M., Zajac-Lamparska, L., Rothen, S., Andryszak, P., Gaspoz, J., ... \& Achab, S. (2021). Internet use and problematic use in seniors: A comparative study in Switzerland and Poland. Frontiers in Psychiatry, 12.

Sadio, A. J., Gbeasor-Komlanvi, F. A., Konu, R. Y., Bakoubayi, A. W., Tchankoni, M. K., Bitty-Anderson, A. M., ... \& Ekouevi, D. K. (2021). Assessment of self-medication practices in the context of the COVID-19 outbreak in Togo. BMC public health, 21(1), 1-9.

Santini, Z. I., Jose, P. E., Cornwell, E. Y., Koyanagi, A., Nielsen, L., Hinrichsen, C., ... \& Koushede, V. (2020). Social disconnectedness, perceived isolation, and symptoms of depression and anxiety among older Americans (NSHAP): a longitudinal mediation analysis. The Lancet Public Health, 5(1), e62-e70.

Souza, Z. A. D., Nemer, C. R. B., Teixeira, E., Neves, A. L. M. D., Nascimento, M. H. M., Medeiros, H. P., ... \& Oliveira, V. L. G. D. (2021). Fatores associados ao enfrentamento da pandemia da COVID-19 por pessoas idosas com comorbidades. Escola Anna Nery, 25.

Rola, M. J. L. (2020). " Solteiras, mas não solteironas..." Perceções, significações e vivências de mulheres solteiras mais velhas.

TOURET, Franck; DE LAMBALLERIE, Xavier. De cloroquina e COVID-19. Pesquisa antiviral , v. 177, p. 104762, 2020.

Machado, A. M. G. (2017). Um debate sobre o envelhecimento populacional e o impacto para o Sistema Único de Saúde (SUS): o perfil de idosos residentes na capital e no interior do estado do Rio Grande do Sul internados em Porto Alegre, 2011-2015.

Velho, F. D., \& Herédia, V. B. M. (2020). O Idoso em Quarentena e o Impacto da Tecnologia em sua Vida/Quarantined Senior Citizens and the Impact of Technology on Their Life. Rosa Dos Ventos-Turismo E Hospitalidade, 12(3).

Vidal, G. P., \& Castro, A. (2020). O Psicodrama clínico on-line: uma conexão possível. Revista Brasileira de Psicodrama, $28(1$ ), 54-64. 